\title{
An improved performance routing protocol based on delay for MANETs in smart cities
}

\author{
Vu Khanh Quy', Pham Minh Chuan', Le Anh Ngoc ${ }^{2}$ \\ ${ }^{1}$ Faculty of Information Technology, Hung Yen University of Technology and Education, Hưng Yên, Vietnam \\ ${ }^{2}$ Swinburne Vietnam, FPT University, Hanoi, Vietnam
}

\begin{tabular}{l} 
Article Info \\
\hline Article history: \\
Received Dec 18, 2020 \\
Revised Jul 16, 2021 \\
Accepted Aug 2, 2021 \\
\hline Keywords: \\
AODV \\
Delay \\
Improve performance \\
Mobile ad hoc networks \\
Routing protocol
\end{tabular}

\begin{abstract}
Mobile ad-hoc networks (MANETs) is a set of mobile devices that can selfconfiguration, self-established parameters to transmission in-network. Although limited inability, MANETs have been applied in many domains to serve humanity in recent years, such as disaster recovery, forest fire, military, intelligent traffic, or IoT ecosystems. Because of the movement of network devices, the system performance is low. In order to MANETs could more contribution in the future of the Internet, the routing is a significant problem to enhance the performance of MANETs. In this work, we proposed a new delay-based protocol aim enhance the system performance, called performance routing protocol based on delay (PRPD). In order to analyze the efficiency of the proposed solution, we compared the proposed protocol with traditional protocols. Experiment results showed that the PRPD protocol improved packet delivery ratio, throughput, and delay compared to the traditional protocols.
\end{abstract}

This is an open access article under the CC BY-SA license.

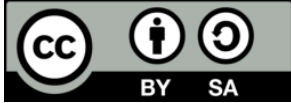

\section{Corresponding Author:}

Le Anh Ngoc

Swinburne Vietnam, FPT University

Hanoi, Vietnam

Email: ngocla2@fe.edu.vn

\section{INTRODUCTION}

The advent of $5 \mathrm{G}$ networks marks a truly digital society. With end-to-end connection speeds for mobile devices reaching $1 \mathrm{Gbps}$, ultra-low latency, extremely high bandwidth, and the ability to connect a huge amount of devices lead to a wide range of services and new application. Besides, according to cisco's prediction, by 2023, global mobile traffic will increase over seven times compared to now, accounting for about $3 / 4$ global network data traffic. Moreover, mobile devices are equipped with the Machine to machine (M2M) modules, which can communicate directly between devices without based on pre-installed infrastructure (the principle of mobile ad-hoc networks (MANETs) formation) [1]. In reality, MANETs was shaped in the 1980s as an organization of mobile radio devices which is self-established and self-configuring parameters to communicate in-network more conveniently [2]. In recent years, MANETs have series of applications for humanity in many areas such as smart agriculture, smart retail, intelligent transportation system, green energy and IoT ecosystems, indicated in [3]-[16]. Figure 1 is an illustration of MANET architecture has eight mobile network nodes.

The MANETs performance depends on the topology, movement speed, and deployment environment. With limited capacity, the MANETs performance is rather low. In MANETs, the mobile network devices must corporate to communicate; routing protocols have a vital role in enhancing performance [17]. Traditional routing protocols such as ad-hoc on-demand distance vector (AODV) and dynamic source routing (DSR) using hop-count metric are not effective [18]. The research and proposal of 
robust, reliable, high-performance routing solutions are critical and interested researchers. Many routing protocols have been introduced in [19]-[23]. However, each solution only fit a specific structure, scenario. Therefore, it is our motivation to study this problem.

Over the years, the performance enhancement research direction for MANETs has been achieved some positive results, based on several different approaches. Accordingly, in the location-based approach, in Zhang et al. [4] proposed the reverse address resolution protocol (RARP) protocol for unmanned aerial vehicles network. The focus of this work aims to predict the location and trajectory of neighbourhood nodes to enhance transmission distance between aerials. Besides, this work also proposed a system structure rapidly updating schema to reducing route re-establishment and latency. Experiment results showed that the proposed protocol significantly enhance the unmanned aerial vehicle network performance. In this direction, Xie and Murase [13] proposed a new geographic location-based routing algorithm called maximum throughput algorithm for optimal position (MTOP). This work focus on determines the lower and upper bounds are derived to determine the search space domain based on feasible location assembles. Besides, it defines a conflict set of locations graph (CSLG) to prove this proposition. Experiment results show that the MTOP algorithm enhanced the system performance compared to the traditional method in other mobility and density MANET scenarios. Bujari et al. [14] proposed a location-based algorithm namely PAB3D for unmanned airborne vehicle (UAV) network which adapted for three-dimensional network scenarios. Experiment results showed that the proposed algorithm enhances the network compared to the existing routing protocol in other density and mobility scenarios.

Accordingly, the radio signal-based approach, Fazio et al. [20] proposed the signal-based routing protocol, which is obtained from MAC for multi-channel MANETs. The focus of this work aims to minimize co-channel noise and enhance system performance. Experiment results showed that the routing protocol enhances the network performance than traditional protocols. Accordingly, in the topology-based approach, Ejmaa et al. [21] proposed a topology-based protocol namely connectivity factor routing protocol (DCFP). The focus of this work proposes the neighbourhood rate-based routing metric. Experiment results have shown that the DCFP protocol enhances the system performance and energy efficiency compared to traditional protocols.

Accordingly, in the traffic-based approach, Quy et al. [22] proposed a new combined-metric-based protocol. The focus of this work offers a new metric, combined from three single metrics, including hops number, link status, and queue, to enhance system performance. Besides, recent studies [23]-[28] also show that the MANETs performance improvement research field in general and the traffic network-based approach is very exciting and attracts great interest from both science and industry.

In this research, we propose a novel delay-based routing protocol by selecting the shortest route with the lowest delay. The rest of this study is organized as follows in section 2, we present related works. Our proposed routing protocol is introduced in section 3 . The performance evaluations and results of the proposed protocol with the traditional protocol for MANETs are presented in section 4, and section 5 is the conclusion.

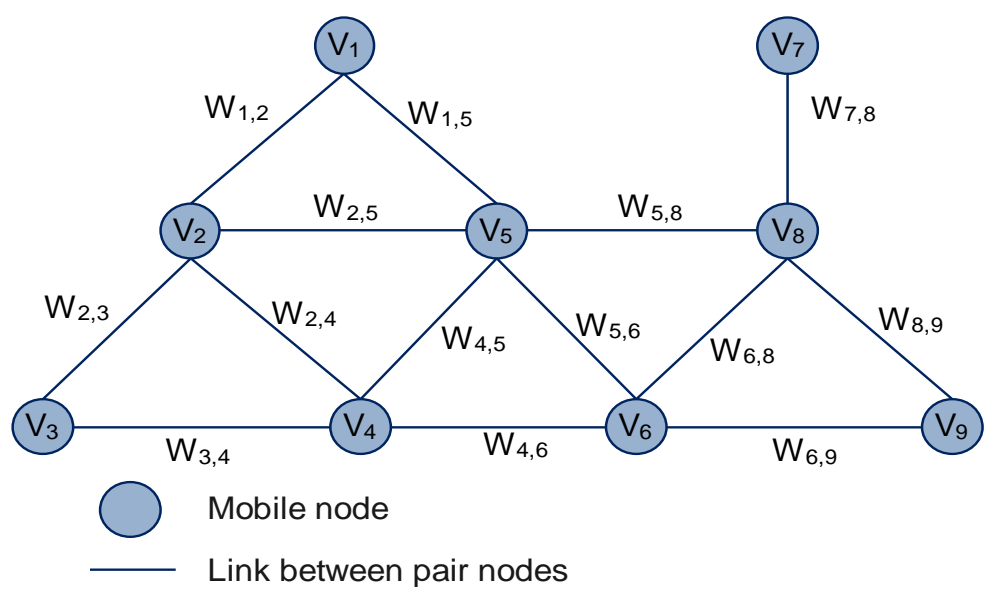

Figure 1. An illustration of the MANET architecture

\section{THE PROPOSED PROTOCOL}

In this section, we will describe the routing parameters, the making-decision function for select the fit route, as well as the operating principle of the proposed protocol. 


\subsection{Protocol description}

The proposed protocol is improved from the AODV protocol, operating on the on-demand principle. When a network node has data that need send, it invokes the route discovery procedure to determine a route to the destination node, Figure 2 . The route discovery procedure broadcasts the route request packets. These packets reach the destination node through intermediate nodes, the red line. The $M_{D}$ node will respond by sending the router reply packet to the $M_{S}$ node, the blue line. Besides the route discovery procedure, the proposed protocol also has route update procedures using the route error packets such, as the yellow line. Finally, the source node receives a candidate route set.

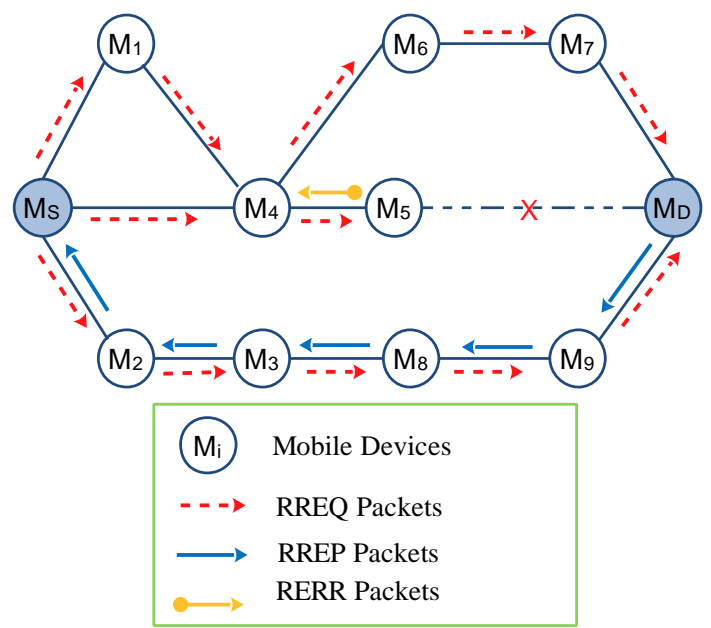

Figure 2. Three operational states of the performance routing protocol based on delay (PRPD) protocol

\subsection{Making-decision function}

After the completed route discovery procedure, the source network node is received the candidate route set, our algorithm defines two that force as follows: the hop numbers (Hopcount) of a candidate path must be within the range [Hopmin, Hopmax]. Paths with hops not within this range will be discarded.

$$
\text { Hopcount }=[\text { Hopmin, Hopmax }]
$$

Where Hopmin, is the minimum distance which packets have to pass from the $M_{S}$ node to the $M_{D}$ node. Aim to decrease the number of candidates, our algorithm defines Hopmax $=$ Hopmin +2 .

In order to purpose select the fit candidate routes and have the lowest delay. Our algorithm defines and uses the concept of the average delay of a path $\left(\right.$ Delay $\left._{i}\right)$. The average delay of a path is the summary delay of all links on that path, determined as in (2):

$$
\operatorname{Delay}_{i}=\operatorname{Total}\left(\operatorname{Delay}_{i}^{1}, \operatorname{Delay}_{i}^{2}, \ldots, \operatorname{Delay}_{i}^{n}\right)
$$

where: Delay ${ }_{i}^{k}$, is the delay of the hop $k^{\text {th }}$ of the route $i$. Let $Z$ is the obtained route number by the (1), and Delay_Set is the delay of the candidate route set, determined by the (2), as (3):

$$
\text { Delay_Set }=\left\{\begin{array}{c}
\text { Delay }_{1} \\
\text { Delay }_{2} \\
\cdot \\
\cdot \\
\cdot \\
\text { Delay }_{Z-1} \\
\text { Delay }_{Z}
\end{array}\right.
$$

The candidate route with the lowest delay can be determined as (4):

$$
\text { Optimalroute }=\operatorname{Min}(\text { Delay_Set })
$$


Accordingly, our optimal route is determined by (4). The details of the routing algorithm are described by pseudocode as shown in Algorithm 1.

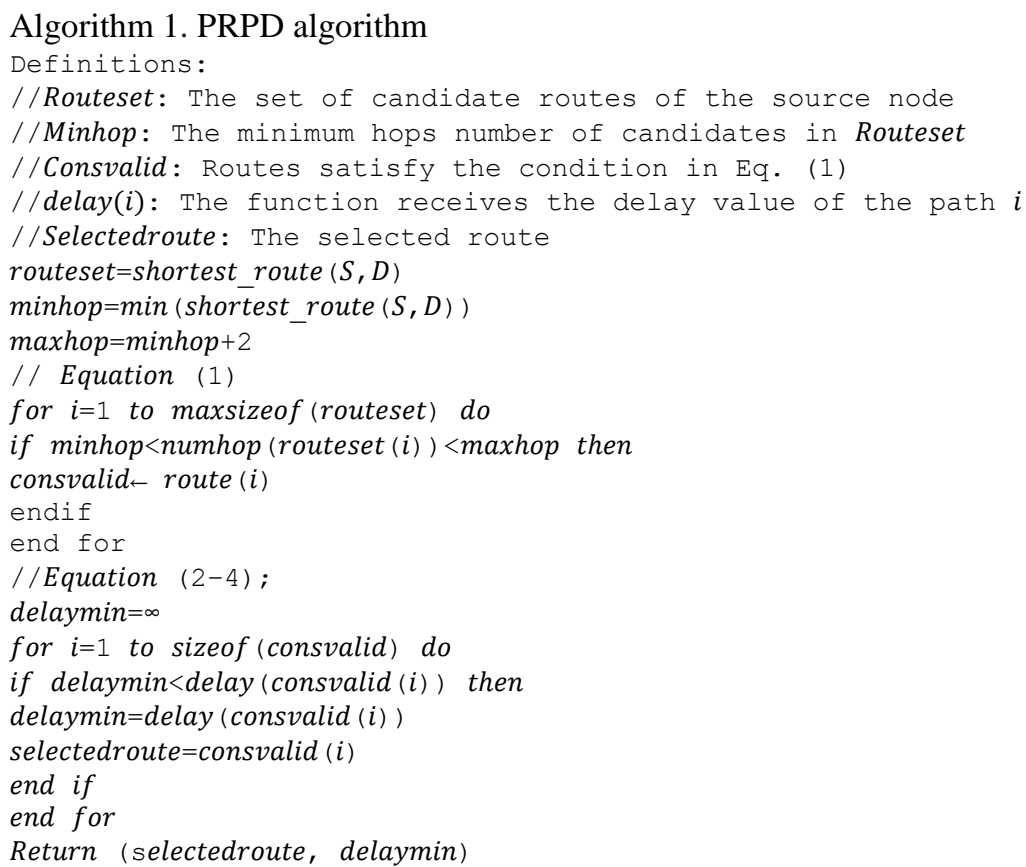

\section{RESULTS AND DISCUSSION}

Aim to the efficiency evaluation of the proposed protocol, this work conducts a simulation system on NS2. Our simulation system includes 250 mobile network nodes, distributed in the range $[1000 \times 1000](\mathrm{m})$. The remaining parameters are shown in Table 1. Our performance routing protocol based on delay (PRPD) protocol is compared to two traditional routing protocols are AODV [29], and DSR [30] in the simulation scenarios under changes on the mobility of the network nodes in the range $[2,4, \ldots, 20](\mathrm{m} / \mathrm{s})$.

Table 1. Simulation parameters

\begin{tabular}{cc}
\hline Parameters & Value \\
\hline Topology size & $1000 \mathrm{~m} \times 1000 \mathrm{~m}$ \\
Number of nodes & 250 \\
Simulation time & $200 \mathrm{~s}$ \\
MAC Layer & 802.11 \\
Traffic type & $\mathrm{CBR}$ \\
Bandwidth & $2 \mathrm{Mbit} / \mathrm{s}$ \\
Transport layer & $\mathrm{UDP}$ \\
Mobile speed & $2-20](\mathrm{m} / \mathrm{s})$ \\
Packet size & $512 \mathrm{byte}$ \\
Transmission range & $250 \mathrm{~m}$ \\
Mobility model & Two-Ray Ground \\
Simulation protocol & PRPD, AODV, DSR \\
\hline
\end{tabular}

\subsection{Performance parameters}

Packet delivery ratio (average PDR) is the percentage ratio of the number of the received package per the sent packages number in a simulation, as (5):

$$
P D R=\frac{P_{s}}{P_{r}} \times 100 \%
$$

End-to-end delay (average delay) is the summerize delay time of all received packets by the destination nodes in a simulation, as in (6):

$$
\text { Delay }_{\text {avg }}=\frac{\sum_{i=1}^{n}\left(t_{r}-t_{s}\right)}{P_{r}}
$$


Throughput: is defined by multiply the received packet number by the destination node and the packet size per one second. This work uses the average throughput concept, denoted is Throughput $_{\mathrm{avg}}$, as in (7):

$$
\text { Throughput }_{\text {avg }}=\frac{P_{r} \times K T}{T \times \text { Delay }_{\text {avg }}}
$$

where:

$P_{r}$ is the total packet numbers received by the destination nodes

$P_{S}$ is the total packet numbers sent by the source node

$t_{r}$ is the time received the packet at the destination device

$t_{s}$ is the time to send the packet from the source device

$T$ is the time of the entire simulation process

$K T$ is the packet size.

\subsection{Results}

Aim to evaluate the efficiency of the PRPD protocol, this work conducted the simulation to compare it with the traditional routing protocols of MANETs as AODV and DSR. This work installs 50 (sourcedestination) pairs in all simulation scenarios. The movement speed of the mobile devices in the range $\left[0, V_{\max }\right]$ with $V_{\max }=[2-20](\mathrm{m} / \mathrm{s})$. The main purpose in changing the movement speed of the mobile devices is to consider the adaptive possibility of the PRPD protocol in the urban-MANET scenarios. Experiment results showed that the PRPD protocol improved significantly the performance metrics compared with the AODV and DSR protocols on the aspect of average latency, average throughput and delivery ratio in different mobility scenarios. When the movement speed of nodes increases, the performance parameters of the PRPD protocol are improved compared to the traditional protocols.

Figure 3 shows the simulation results based on the throughput parameter. Observe results showed that the throughput of the PRPD is enhanced compared to the AODV and DSR protocols. Results demonstrated the PRPD protocol can work well in the quality of service requirement multimedia-MANET applications.

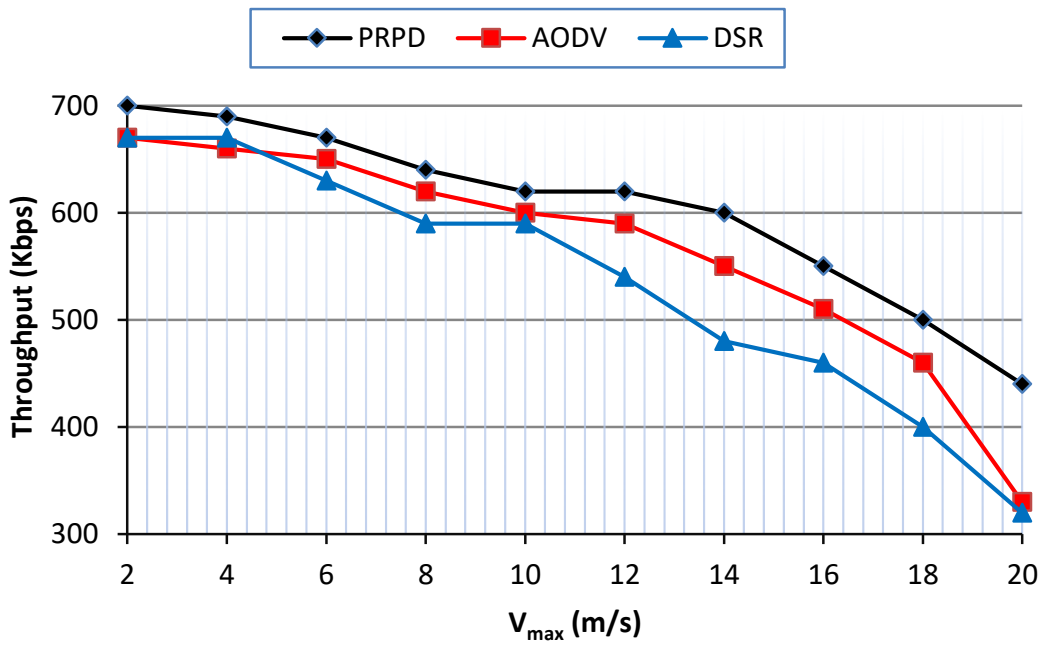

Figure 3. Average throughput with velocity

Figure 4 presents the simulation results based on the latency time parameter. When the movement speed of nodes increase, the latency time parameter of protocols tends to increase. At velocity $V_{\max }=20(\mathrm{~m} / \mathrm{s})$, equivalent to the average velocity is $72(\mathrm{~km} / \mathrm{h})$, the average delay of all protocols are significantly high, the average delay of the AODV and DSR protocols are reaching about 0.9 (s), while the average delay of the PRPD is about 0.8 (s). The experience results showed that, although protocols are improved the network performance. However, the higher performance routing solutions need to be further researched for different mobility urban-MANET scenarios. 


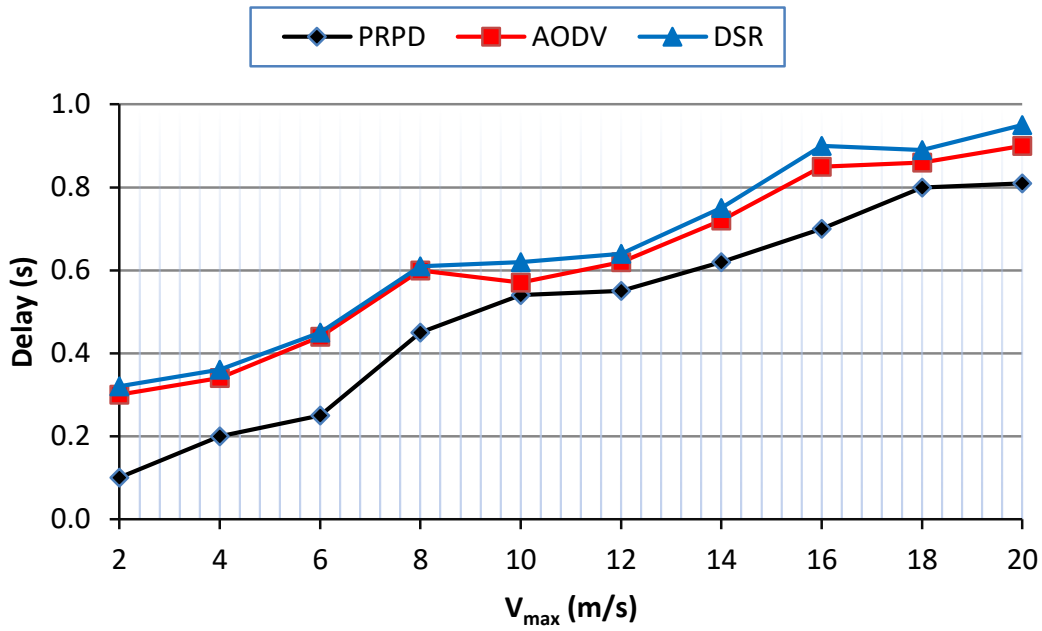

Figure 4. Average delay with velocity

Figure 5 presents the simulation results based on the average PDR. Simulation results show that when $V_{\max } \leq 6(\mathrm{~m} / \mathrm{s})$, the average PDR of protocols is significantly high, reach about $95 \%$. However, the average RDP of protocols decreases rapidly when the movement speed of nodes increases. All simulation resulted shown the efficiency of the proposed protocol compared to the traditional protocols for the different mobility multimedia-MANET scenarios. The results also showed that with the same mobility scenarios, the PRPD protocol improved the performance parameters compared to the traditional protocols such as AODV and DSR. In order to more demonstrate the adaptive of the PRPD protocol, it should be considered under the different changes of the network traffic. The detail of this problem will be presented in our future studies.

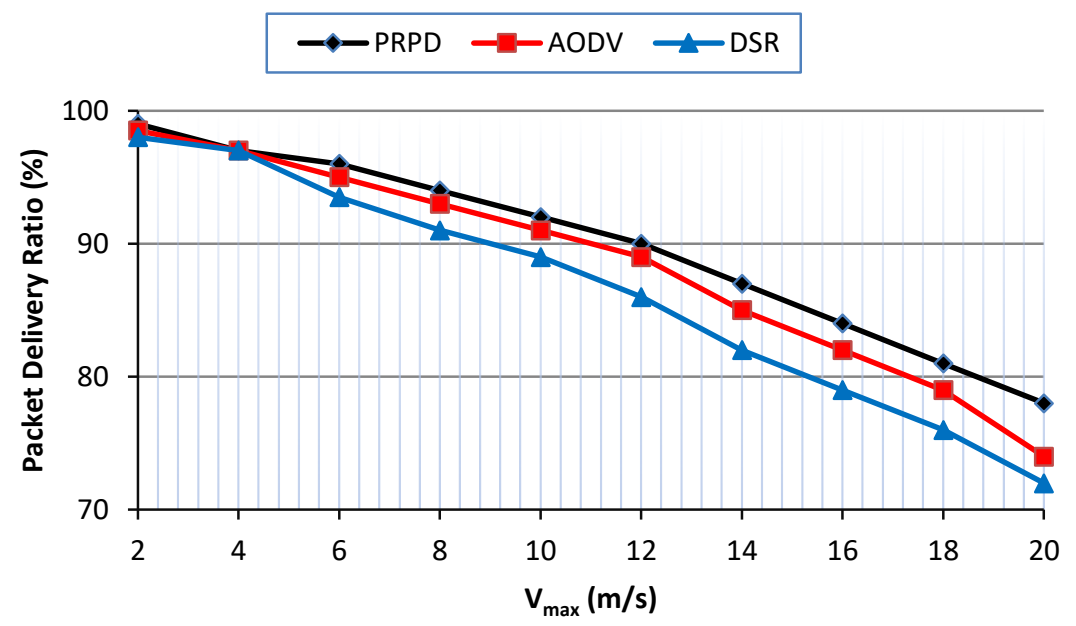

Figure 5. Average PDR with velocity

\section{CONCLUSION}

In this paper, we proposed a delay-based routing protocol aim enhance performance for the multimedia-MANET scenarios called PRPD. The focus of this work offered a multi-metrics-based route selection method. The first, the candidate route must meet the hop number condition, and the second, the candidate with the lowest route average delay will be selected. Aim more demonstrate the adaptive of the PRPD protocol, this work installed the simulation scenarios under the mobility changes. Observe results showed that the PRPD protocol enhances significantly network performance on aspect of average delay, average through, and average PDR compared to traditional AODV and DSR routing protocols. We will focus on the PRPD protocol performance evaluation under changes in the velocity and different error rates in the further studies. 


\section{REFERENCES}

[1] Q. K. Vu, N. V. Hoai, and L. D. Manh, "A survey of state-of-the-art energy efficiency routing protocols for MANET," International Journal of Interactive Mobile Technologies, vol. 14, no. 9, pp. 215-226, 2020, doi: 10.3991/ijim.v14i09.13939.

[2] S. Laqtib, K. El Yassini, and M. L. Hasnanoui, "A technical review and comparative analysis of machine learning techniques for intrusion detection systems in MANET," International Journal of Electrical and Computer Engineering (IJECE), vol. 10, no. 3, pp. 2701-2709, 2020, doi: 10.11591/ijece.v10i3.pp2701-2709.

[3] V. K. Quy, V. H. Nam, D. M. Linh, N. T. Ban, and N. D. Han, "A survey of QoS-aware routing protocols for the MANET-WSN convergence scenarios in IoT networks," Wireless Personal Communications, 2021, doi: 10.1007/s11277-021-08433-z.

[4] A. Zhang, L. Wang, X. Ye, and X. Lin, "Light-weight and robust security-aware D2D-assist data transmission protocol for mobile-health systems," in IEEE Transactions on Information Forensics and Security, vol. 12, no. 3, pp. 662-675, Mar. 2017, doi: 10.1109/TIFS.2016.2631950.

[5] F. Parvaresh and F. Faghani, "A new routing algorithm based on 2-hop neighbourhood information scheme for total power minimisation in wireless ad hoc networks," International Journal of Systems, Control and Communications, vol. 12, no. 2, pp. 91-115, 2021, doi: 10.1504/IJSCC.2021.114597.

[6] B. Ojetunde, N. Shibata, and J. Gao, "Secure payment system utilizing MANET for disaster areas," in IEEE Transactions on Systems, Man, and Cybernetics: Systems, vol. 49, no. 12, pp. 2651-2663, Dec. 2019, doi: 10.1109/TSMC.2017.2752203.

[7] W. Castellanos, J. C. Guerri, and P. Arce, "Performance evaluation of scalable video streaming in mobile ad hoc networks," in IEEE Latin America Transactions, vol. 14, no. 1, pp. 122-129, Jan. 2016, doi: 10.1109/TLA.2016.7430071.

[8] P. Bellavista, A. Corradi, and L. Foschini, "Self-organizing seamless multimedia streaming in dense manets," in IEEE Pervasive Computing, vol. 12, no. 1, pp. 68-78, Jan.-Mar. 2013, doi: 10.1109/MPRV.2012.22.

[9] Y. Chen, C. Hu, E. H. Wu, S. Chuang, and G. Chen, "A delay-sensitive multicast protocol for network capacity enhancement in multirate MANETs," in IEEE Systems Journal, vol. 12, no. 1, pp. 926-937, Mar. 2018, doi: 10.1109/JSYST.2017.2677952.

[10] P. J. Nicholas and K. L. Hoffman, "Optimal channel assignment for military MANET using integer optimization and constraint programming," 2016 IEEE Military Communications Conference, MILCOM-2016, 2016, pp. 1114-1120, doi: 10.1109/MILCOM.2016.7795479.

[11] S. Rosati, K. Krużelecki, G. Heitz, D. Floreano, and B. Rimoldi, "Dynamic routing for flying ad hoc networks," in IEEE Transactions on Vehicular Technology, vol. 65, no. 3, pp. 1690-1700, Mar. 2016, doi: 10.1109/TVT.2015.2414819.

[12] V. K. Quy, N. T. Ban, V. H. Nam, D. M. Tuan, and N. D. Han, "Survey of recent routing metrics and protocols for mobile ad-hoc networks," Journal of Communications, vol. 14, no. 2, pp. 110-120, 2019, doi: 10.12720/jcm.14.2.110-120.

[13] J. Xie and T. Murase, "An optimal location allocation by multi-user cooperative mobility for maximizing throughput in MANETs," in IEEE Access, vol. 8, pp. 226089-226107, 2020, doi: 10.1109/ACCESS.2020.3044886.

[14] A. Bujari, C. E. Palazzi, and D. Ronzani, "A comparison of stateless position-based packet routing algorithms for FANETs," in IEEE Transactions on Mobile Computing, vol. 17, no. 11, pp. 2468-2482, Nov. 2018, doi: 10.1109/TMC.2018.2811490.

[15] A. M. Mezher and M. A. Igartua, "Multimedia multimetric map-aware routing protocol to send video-reporting messages over VANETs in smart cities," in IEEE Transactions on Vehicular Technology, vol. 66, no. 12, pp. 10611-10625, Dec. 2017, doi: 10.1109/TVT.2017.2715719.

[16] S. Mumtaz, A. Bo, A. Al-Dulaimi, and K. Tsang, "Guest editorial 5G and beyond mobile technologies and applications for industrial IoT (IIoT)," in IEEE Transactions on Industrial Informatics, vol. 14, no. 6, pp. 2588-2591, Jun. 2018, doi: 10.1109/TII.2018.2823311.

[17] S. S. Mohamed, A. I. Abdel-Fatah, and M. A. Mohamed, "performance evaluation of MANET routing protocols based on QoS and energy parameters," International Journal of Electrical and Computer Engineering, vol. 10, no. 4, pp. 3635-3642, 2020, doi: 10.11591/ijece.v10i4.pp3635-3642.

[18] D. J. Persis and T. P. Robert, "Review of ad-hoc on-demand distance vector protocol and its swarm intelligent variants for mobile ad-hoc network," IET Networks, vol. 6, no. 5, pp. 87-93, 2017, doi: 10.1049/iet-net.2017.0015.

[19] G. Gankhuyag, A. P. Shrestha, and S. Yoo, "Robust and reliable predictive routing strategy for flying ad-hoc networks," in IEEE Access, vol. 5, pp. 643-654, 2017, doi: 10.1109/ACCESS.2017.2647817.

[20] P. Fazio, F. De Rango, and C. Sottile, "A predictive cross-layered interference management in a multichannel MAC with reactive routing in VANET," in IEEE Transactions on Mobile Computing, vol. 15, no. 8, pp. 1850-1862, Aug. 2016, doi: 10.1109/TMC.2015.2465384

[21] A. M. E. Ejmaa, S. Subramaniam, Z. A. Zukarnain, and Z. M. Hanapi, "Neighbor-based dynamic connectivity factor routing protocol for mobile ad hoc network," in IEEE Access, vol. 4, pp. 8053-8064, 2016, doi: 10.1109/ACCESS.2016.2623238

[22] V. K. Quy, N. T. Ban, and N. D. Han, "A multi-metric routing protocol to improve the achievable performance of mobile Ad Hoc networks," Studies in Computational Intelligence, vol. 769, pp. 445-453, 2018, doi: 10.1007/978-3-319-76081-0_38.

[23] A. Abuashour and M. Kadoch, "Performance improvement of cluster-based routing protocol in VANET," in IEEE Access, vol. 5, pp. 15354-15371, 2017, doi: 10.1109/ACCESS.2017.2733380.

[24] C. K. Toh, A. Le, and Y. Cho, "Load balanced routing protocols for ad hoc mobile wireless networks," in IEEE Communications Magazine, vol. 47, no. 8, pp. 78-84, Aug. 2009, doi: 10.1109/MCOM.2009.5181896.

[25] O. O. Khalifa, D. E. M. Ahmed, and A. H. A. Hashim, "Video streaming over ad hoc on-demand distance vector routing protocol," Bulletin of Electrical Engineering and Informatics (BEEI), vol. 8, no. 3, pp. 863-874, 2019, doi: 10.11591/eei.v8i3.1510.

[26] M. H. Hassan et al., "Mobile ad-hoc network routing protocols of time-critical events for search and rescue missions," Bulletin of Electrical Engineering and Informatics (BEEI), vol. 10, no. 1, pp. 192-199, 2021, doi: 10.11591/eei.v10i1.2506.

[27] Y. Khamayseh, M. B. Yassein, and M. Abu-Jazoh., "Intelligent black hole detection in mobile AdHoc networks," International Journal of Electrical and Computer Engineering (IJECE), vol. 9, no. 3, pp. 1968-1977, 2019, doi: 10.11591/ijece.v9i3.pp19681977.

[28] M. Abdelhaq et al., "The resistance of routing protocols against DDOS attack in MANET," International Journal of Electrical and Computer Engineering (IJECE), vol. 10, no. 5, pp. 4844-4852, 2020, doi: 10.11591/ijece.v10i5.pp4844-4852.

[29] “RFC3561.” IETF. [Online]. Available: https://www.ietf.org/rfc/rfc3561 (accessed Feb. 10, 2021).

[30] “RFC4728.” IETF. [Online]. Available: https://www.ietf.org/rfc/rfc4728 (accessed Feb. 10, 2021). 